\title{
SPECTROPHOTOMETRY OF COMET KOHOUTEK (1973f) DURING PRE-PERIHELION PERIOD
}

\author{
G. S. D. Babu
}

\section{INSTRUMENTATION AND OBSERVATIONS}

A total of eleven scans of the head of comet Kohoutek (1973f) were obtained during the pre-perihelion period with a photoelectric spectrum scanner (Babu, 1971), mounted at the Nasmyth focus (f/13.1) of the 52-cm telescope, on 7,10 , 11, 13, 14 and 15 December 1973 at the rate of two scans per day, except on 7 December when only one scan was taken. The observational procedure was similar to that for comet Bennett (1969 i) (Babu and Saxena, 1972), excepting that a diaphragm of $3 \mathrm{~mm}$ diameter equivalent of $93^{\prime \prime}$ of the sky was used at the entrance of the monochromator. The width of the exit slit was equivalent to a spectral window of $3.5 \mathrm{~nm}$. The scans covered the spectral range from 370 to $640 \mathrm{~nm}$. The basic data of the comet during the period of observations is given in Table I.

In addition, two late type stars, $\pi$ Hya (K2 III) and $\beta \mathrm{Crv}$ (G5 III) were observed on each night to serve as comparison stars. Since $\beta \mathrm{Crv}$ is suspected to be a variable (Hoffleit, 1964), $\pi$ Hya has been chosen as the comparison star.

The reduction technique is same as that used in the case of comet Bennett (loc. cit.) where all the measurements were normalised to $479 \mathrm{~nm}$. In order to get the normalised relative magnitudes of the comet at each wavelength, the 


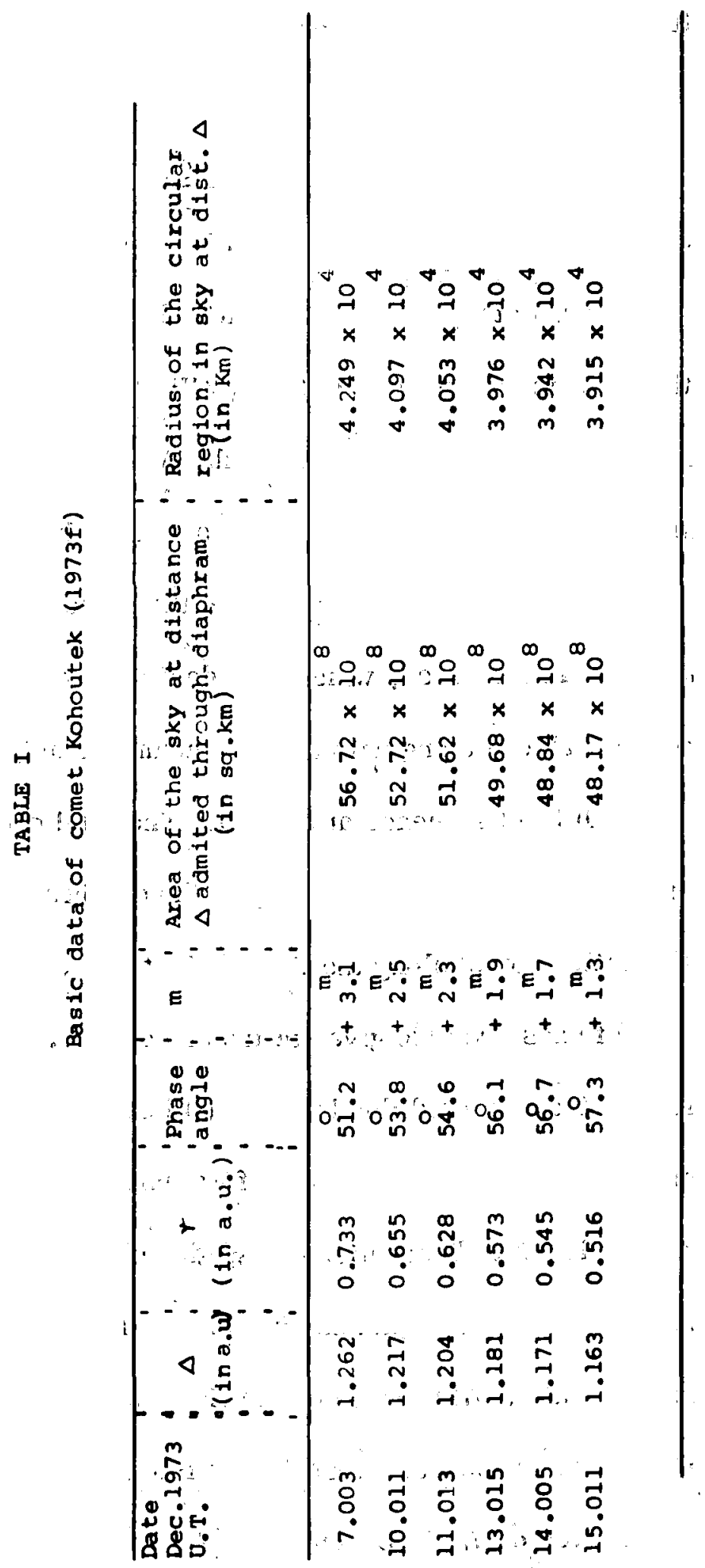


differential magnitudes ( comet- $\pi$ Hya) $\lambda$ were obtained and then converted into intensities.

\section{RELATIVE FLUX DISTRIBUTIONS}

Figure 1 shows the mean relative flux distributions of the head of comet Kohoutek (1973 f) on various dates, the vertical lines showing the probable errors. In these the emission features of $\mathrm{CN}, \mathrm{C}_{3}, \mathrm{CH}$, the principal Swan band sequences of $\mathrm{C}_{2}$ and $\mathrm{Na}$ have readily been identified. After locating the continuum in the scans, (Swings and Haser, 1956), the areas of the emission band profiles were planimetered. The probable errors in these measurements are found to be less than $5 \%$. These areas, which are identified with the total band intensities, are given in Table II along with the continuum intensities at $479 \mathrm{~nm}$ relative to the $C_{2}(\Delta V=0)$ band sequence at $516 \mathrm{~nm}$. The total energies streaming out of a cylinder with diameter corresponding to the diaphragm size and extending through the entire comet along the line of sight in $\mathrm{C}_{2}(\Delta \mathrm{V}=0)$ band along with the observed fluxes are also given in the same table. The reduction was made by multiplying the observed flux by $4 \pi \Delta^{2}$ (O'Dell and Osterbrock, 1962).

It is clear from this table that the intensity of Na emission steadily increased with the decreasing $r$ during our observational period. This is similar to the case of comet Ikeya-Seki (1965 f) (Bappu and Sivaraman, 1967). However, it has been reported by Bappu et al. (1973) that in comet Kohoutek (1973 f) the Na emission was first noticed on 8.98 December 1973. But our observations show that Na was in emission even on 7.003 December 1973 , though very faintly. 


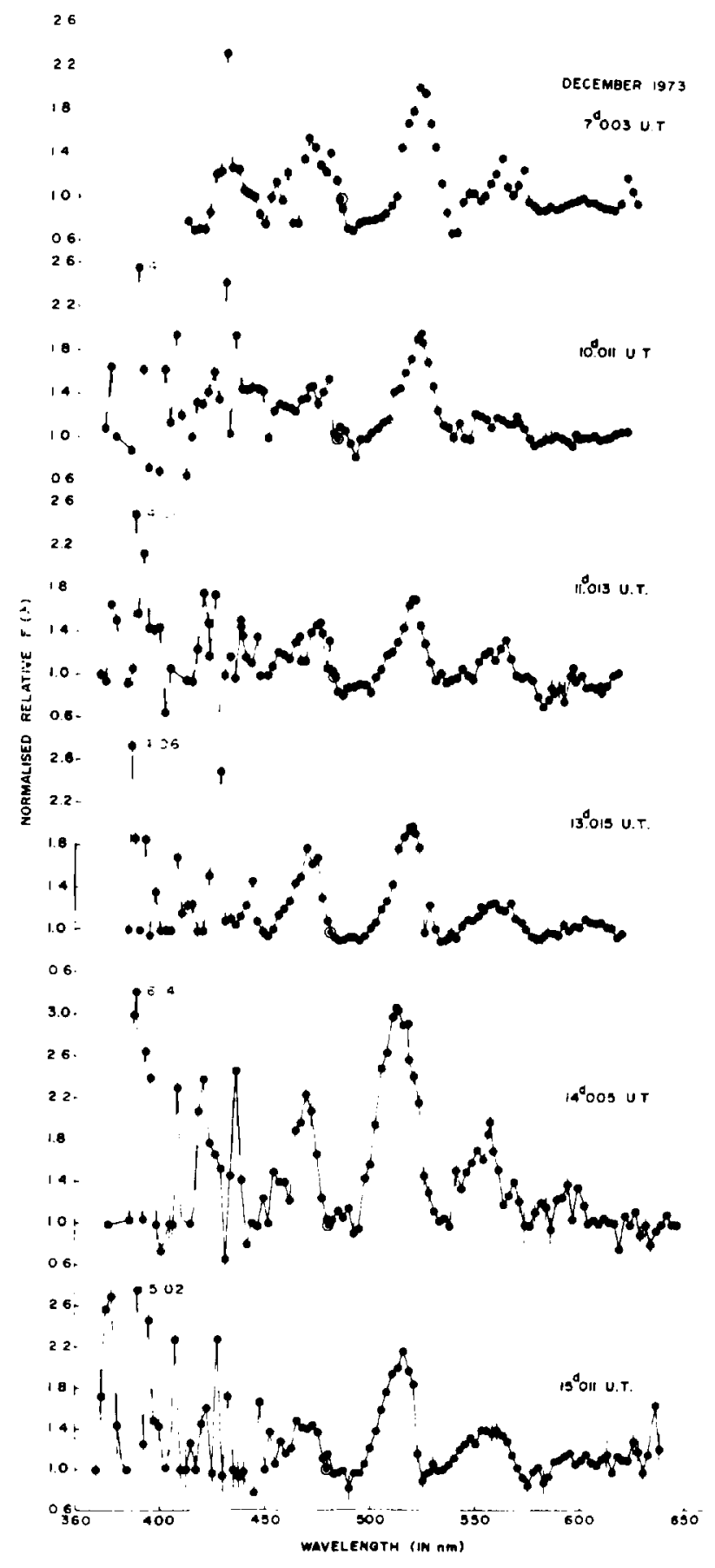

Figure 1: Relative flux distributions of the head of comet Kohoutek (1973 f) on various dates normalised to $\lambda 479 \mathrm{~nm}$. The normalisation point is shown as a circle with a dot. Intensity at $\mathrm{CN}(\lambda 388 \mathrm{~nm})$ is reduced to fit the plot and is given by the numbers adjacent to the peak. The vertical lines denote the limits of errors. 


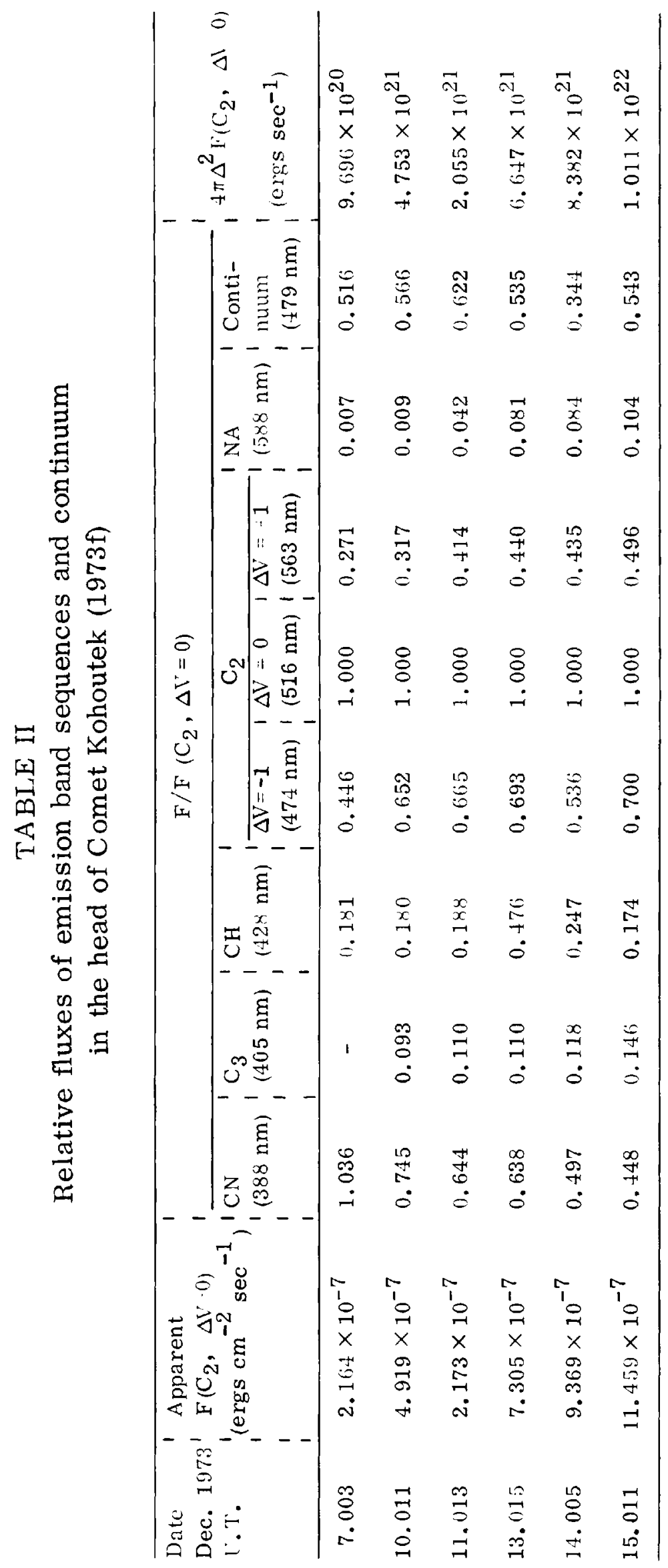


Dobrovolskii (1961) has pointed out that the emissions due to $\mathrm{C}_{2}$ and $\mathrm{C}_{3}$ are less dependent on $r$ when $r<1.5$ a. u. . Since all our observations are obtained when $r$ was less than 1.0 a. $\mathrm{u}_{\bullet}$, the fluxes due to these two homonuclear molecules are expected to be nearly constant as $r$ decreased. But, in the present case, the absolute flux of $\mathrm{C}_{2}$ emission at $516 \mathrm{~nm}$ and even the relative fluxes of $\mathrm{C}_{2}$ emissions at $474 \mathrm{~nm}$ and at $563 \mathrm{~nm}$ show an increase with the decrease in $r$. Similar is the case with the $C_{3}$ emission at $405 \mathrm{~nm}$. This is akin to the case of comet Rudnicki (1966 e) (Mayer and O'Dell, 1968). This increase in the fluxes can be explained only in terms of an increase in the abundances of $\mathrm{C}_{2}$ and $\mathrm{C}_{3}$ in the head of the comet due to some internal processes which depend on $r$.

The relative intensity in the $\mathrm{CN}$ band at $388 \mathrm{~nm}$ shows a steady decrease with decreasing $r$. This behaviour matches that of comet Bester (1947 k) (vide Swings, 1965).

The relative continuum intensities at $479 \mathrm{~nm}$ are weaker than those found in comet Ikeya-Seki (1967 n), Honda (1968 c) and Thomas 1968 b) as given by Gebel (1970). Even the relative continuum intensities of comet Bennett (1969 i) (vide Babu and Saxena, 1972) were stronger than the present values. Nevertheless comet Kohoutek (1973 f) is not void of continuum, thereby showing the presence of dust particles. 


\section{CONT INUUM ENERGY DISTRIBUTIONS}

The continuum energy distributions in the head of comet Kohoutek (1973 f) on different dates along with those of $\pi$ Hya and $\beta \mathrm{Crv}$ have been obtained independently, using $\gamma$ Gem as a standard star. The calibration of $\gamma$ Gem was taken from Wolff et al. (1968). The adopted monochromatic values relative to 479 $\mathrm{nm}$ are given in Table III and are plotted in Figure 2. The energy distribution of the sun (Arpigny, 1965) is also plotted in the same figure.

On 7 December, when the phase angle was about $51^{\circ} .2$, the energy curve of the comet is found to fall around midway between those of $\beta \mathrm{CrV}$ (G5 III) and the Sun (G2 V). On the later dates, as the phase angle increased, the energy curves of the comet approached that of Sun. During the last three days of our observations, they were found to nearly coincide with that of the Sun. It may be noted here that the phase angle of $57^{\circ} .3$ on 15 December was very close to the preperihelion maximum value of $57^{\circ} .8$ on 17 December. Thus the reddening of the scattered light coming from the head of the comet decreased as the phase angle increased, relative to the energy curve of sun, which compares with the case of Comet Bennett (Babu and Saxena, 1972). However, we have no usable observations available to us to study if this trend continued or not with comet Kohoutek after the comet went past its maximum phase angle on 17 December. 


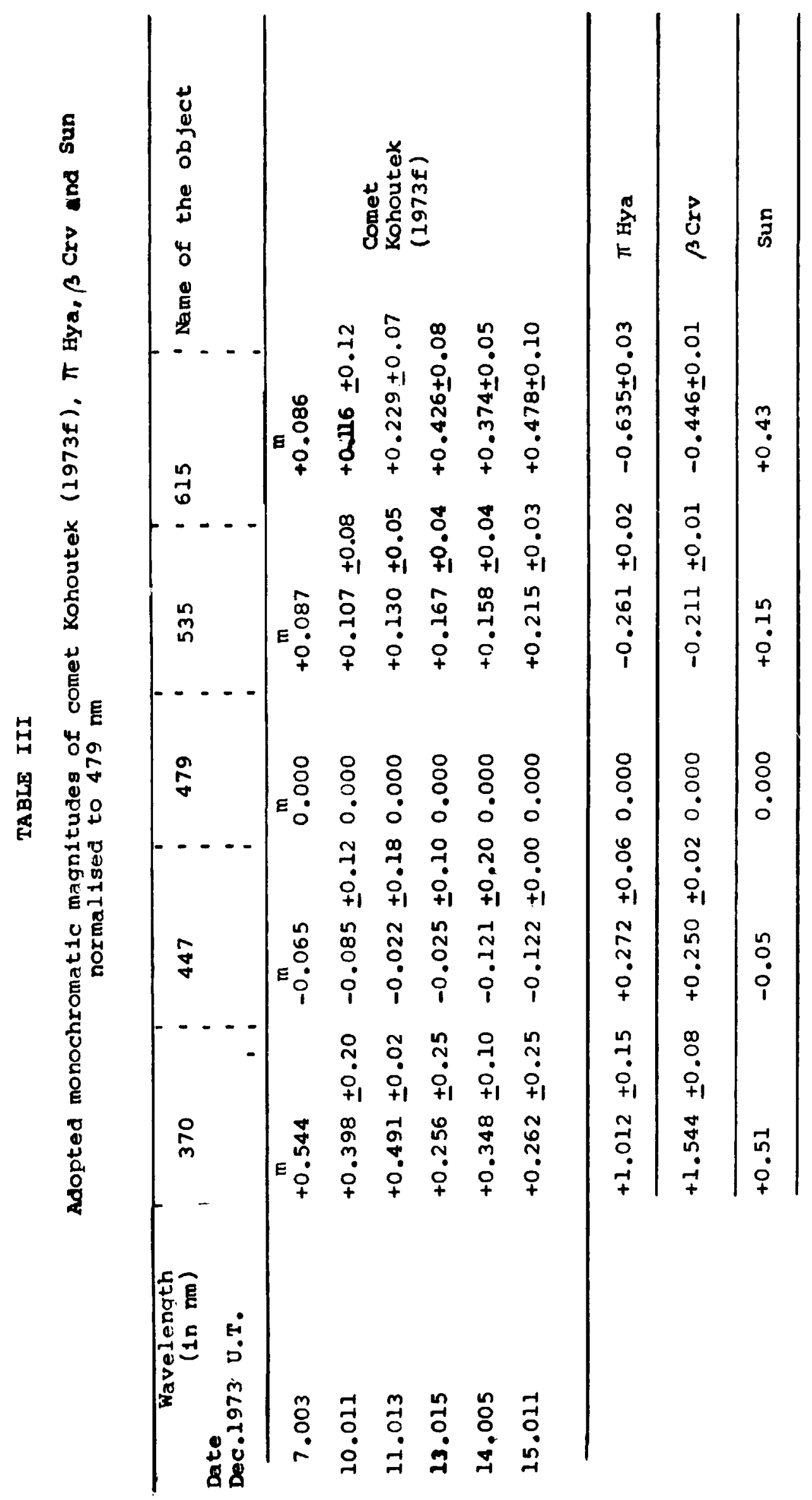




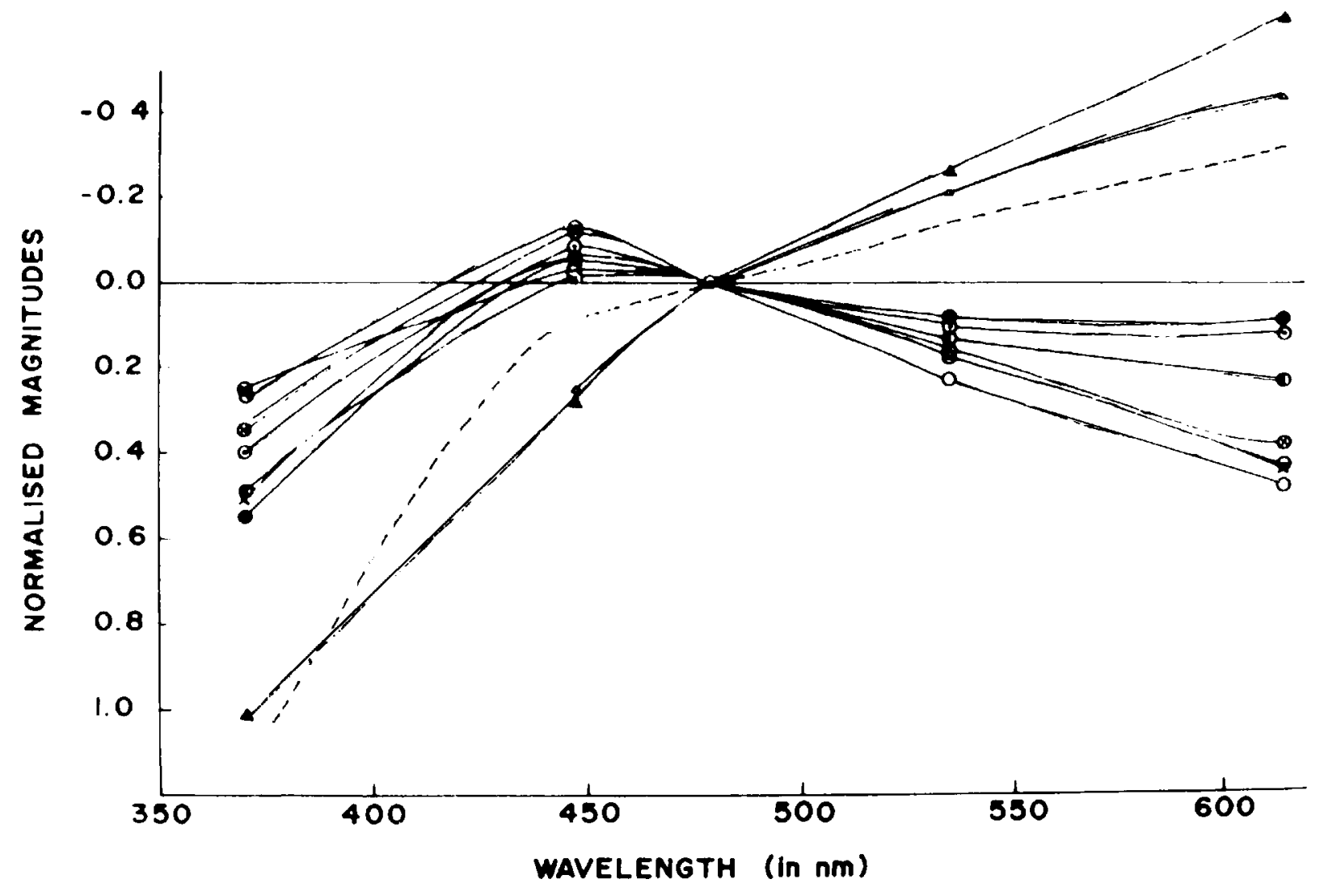

Figure 2: Continuum energy distribution curves of the head of comet Kohoutek (1973 f), which are denoted by circles in the following manner:

- for 7.003 December, $\odot$ for 10.011 December, $\odot$ for 11.013 December, $\Theta$ for 13.015 December, $\otimes$ for 14.005 December and $O$ for 15.011 December, 1973, compared with those of $\pi$ Hya $(\boldsymbol{\Delta}), \beta \mathrm{Crv}(\Delta)$, Sun $(\mathrm{X})$ and solar light scattered according to $\lambda^{2}$ law (-----). All curves are normalised to $479 \mathrm{~nm}$ and the Balmer discontinuity is smoothed out. 
In the previous studies, however, some comets are known to have had a pure reflection continuum, in the sense that the continuum spectra were unreddened with respect to that of the Sun (Arpigny, 1965; Gebel, 1970), whereas some others were found to produce reddened scattered continuous spectra matching those of late type stars around G8 (Bappu and Sinvhal, 1960; Kharitonov and Rebristyi, 1974; Liller, 1960; Vanýsek, 1960; Walker, 1958).

Arpigny (1965) has pointed that the dust grains in comets possibly have a certain range of sizes and the predominant size could be different in different comets. This could produce both reddened and unreddened scattered continuous spectra ir the respective comets. Also, assuming dielectric particles and a narrow distribution function of sizes, the selectivity of scattered light depends strongly but not monotonously on the phase angle*. But, it is not known whether the size distribution function and the predominant particle size are varying or not in a given comet along its path around the sun.

To find out the effect due to the variations in the physical parameters only on the intensity in a particular wavelength, the phase angle effect must be separated out from the total. We believe, in principle, it is possible to use appropriate scattering functions for various size distributions of particles and find out the variation of the intensity at a particular wavelength in a given phase angle. This being a complicated program, the same has not yet been attempted.

\footnotetext{
* The author is thankful to Prof. Vanýsek for bringing this to his notice.
} 


\section{ACKNOWLEDGMENTS}

The author is grateful to Dr. D. S. Sinvhal for suggesting the problem and for useful discussions and suggestions. Thanks are also due to Dr. M. C. Pande for his critical comments, to Dr. B. G. Marsden for sending the computer printout of the ephemeris of the comet under study and Mr. U. C. Joshi for his participation in the observations at the telescope. 


\section{REFERENCES}

1. Arpigny, C., 1965, Liege Inst. Astrophys. Reprint No. 493.

2. Babu, G. S.D., 1971, Observatory, 91, 115.

3. Babu, G.S.D. and Saxena, P.P., 1972, Bull. Astron. Inst. Czech., 23, 346.

4. Bappu, M. K. V. and Sinvhal, S. D., 1960, Monthly Notices Roy. Astron. Society, 120, 152.

5. Bappu, M.K. V. and Sivaraman, K.R., 1967, Kodaikanal Obs. Bull. No. 178.

6. Bappu, M.K. V., Parthasarathy, M. and Sivaraman, K.R., 1973, IAU Cir. No. 2608.

7. Dobrovolskii, O. B., 1961, Akad. Tadj. SSR, VII.

8. Gebel, W. L., 1970, Astrophys. J. , 161, 765.

9. Hoffleit, D., 1964, Cat. Bright Stars, Yale Uni. Obs., New Haven, Connecticut.

10. Kharitonov, A. V. and Rebristyi, V.T., 1974, Sov, Astron., 17, 672.

11. Liller, W., 1960, Astrophys. J., 132, 867.

12. Mayer, P. and O'Dell, C.R., 1968, Astrophys. J., 153, 951.

13. O'Dell, C.R. and Osterbrock, D. E., 1962, Astrophys. J., 136, 559.

14. Swings, P., 1965, Quart. Journ. Roy. Astron. Soc., 6, 28.

15. Swings, P. and Haser, L., 1956, Atlas of Representative Cometary Spectra (Louvain: Ceuterick Press), p. 11.

16. Vanýsek, V., 1960, Bull. Astron. Inst. Czech., 11, 215.

17. Walker, M.F., 1958, Publ. Astron. Soc. Pacific, 70, 191. 